\title{
II. \\ Beiträge \\ zur Interpretation von Scävolas Digesten II.
}

\author{
Von \\ Herrn Geheimerath Professor Dr. Th. Schirmer \\ in Königsberg.
}

1. L. $122 \S 5$ D. V. O. 45, 1. Seia heres unius tutoris, cum herede pupillae transactione pacto solo facta, maiorem partem solvit, residuam cavit: sed ilico negavit heres se transactionem servare et apud iudicem tutelae egit et victus provocavit ad competentem iudicem et $a b$ eo quoque ad principem idem provocavit, et iniusta haec quoque provocatio eius pronuntiata est. quaesitum est, cum per heredem pupillae mora intercesserit, quominus pecunia in stipulationem deducta $a b$ herede tutoris solveretur, nec unquam petierit, an ei hodie debeantur usurae ab herede tutoris. respondit, si Seia non cessasset ex stipulatione pecuniam offerre, iure usuras non deberi. (Scaevola lib. 28 Dig.)

In der vorliegenden Stelle springt eine Schwierigkeit sofort in die Augen; die Vormundschaftsklage ist von dem Erben der Mündelin nutzlos verbraucht, der Process bis in die höchste Instanz gediehen und dort mit Rücksicht auf den zwischen den Parteien abgeschlossenen Vergleich zu Ungunsten des Klägers entschieden worden. Ihm bleibt also einzig die Klage aus der Stipulation auf die Zahlung der versprochenen Restsumme. Wie kann nun aber dabei die Leistung von Verzugszinsen in Frage kommen, welche officio iudicis praestantur, also nur in bonae fidei iudiciis gefordert werden können? - Man hat sich deshalb von frühen Zeiten her zu der Annahme gedrängt gesehen, die Stipulation habe direct oder indirect die Zinsverpflichtung des Schuldners mit umfasst. Noch 
F. Mommsen meint in seinen Beiträgen zum Obligationenrecht Bd. III $\S 24$ Anm. 10, dass, ,die Stipulation wohl auf dasjenige lautete, was die Seia ex causa transactionis noch zu leisten hatte. Man müsste sonst annehmen, dass in dem zur Entscheidung stehenden Fall ausdrücklich, aber nur für den Fall der Mora, Zinsen versprochen seien". Dies letztere war bekanntlich die bei den Aelteren herrschende Meinung, vgl. Glück, Comm. Bd. IV p. 419 Anm. 67. Es ist begreiflich, wenn Mommsen in derselben nur einen äussersten Nothbehelf erblicken will, denn wie darf man dem Juristen die Ungereimtheit zutrauen, er habe bei seiner Angabe des Inhalts der Stipulation gerade das für die aufgeworfene Frage Wesentliche, die ausdrückliche Verheissung von Zinsen im Falle eines Verzuges einfach weggelassen. Aber auch Mommsens eigene Auffassung ist mit dem Wortlaute des Fragmentes nicht in Einklang zu bringen; maiorem partem solvit, residuam cavit, heisst es da. Wie die maior pars, welche gezahlt ist, nur eine bestimmte Geldsumme sein kann, so lässt sich auch unter dem residuam cavit nur die Stipulation einer ebensolchen Geldsumme verstehen. Zudem, ein quidquid ex transactione dare facere oportet ist bei dem formlosen Abschlusse des Vergleichs streng genommen gar nicht einmal vorhanden, die Fassung der Stipulation müsste also auf quanti ea res est oder quanti interest gelautet haben, was mit dem einen Worte „residua" um so weniger angedeutet werden durfte, je unwillkürlicher dabei ein Jeder zunächst an eine bestimmte Summe denken musste. Vergessen wir doch nicht, Scävola ist gerade in der knappen und correcten Darlegung der dem Rechtsfall unterliegenden Thatsachen ein unübertroffener Meister, und hier sollte er sich nun so zweideutig und irreleitend wie möglich ausgedrückt haben! Sonach wird kaum etwas anderes übrig bleiben, als die Leistung der Verzugszinsen mit Cujacius (in den Noten zum Titel de verbor. obl. bei unserem Paragraphen) in Verbindung mit der Klage aus der Vormundschaft $z u$ bringen.

Allein wie kann aus dem bereits durch sämmtliche Instanzen hindurch gezogenen Processe noch ein Urtheil in Aussicht stehen? Wie ich meine, bietet uns unser Text dafür allerdings einen gewissen Anhalt. Als Gegenstand des Streites 
zwischen den Parteien wird uns ausschliesslich die rechtsverbindliche Kraft des Vergleiches genannt; nur darüber, so darf man schliessen, sind die von Scävola erwähnten, wiederholten Entscheidungen ergangen. Es handelt sich also um ein in dem Rechtsstreite gefälltes Zwischenurtheil. Dass solche Zwischenurtheile appellabel waren, wenigstens $\mathrm{zu}$ - Scävolas Zeiten, ersehen wir aus

L. 39 D. de minor. 4, 4. Intra utile tempus restitutionis apud praesidem petierunt in integrum restitutionem minores et de aetate sua probaverunt: dicta pro aetate sententia adversarii, ut impedirent cognitionem praesidis, ad imperatorem appellaverunt: praeses in eventum appellationis cetera cognitionis distulit. (Scaevola lib. 2 Dig.)

Also nur bezüglich des Alters der die Restitution Verlangenden hat der Statthalter erkannt, alles andere bleibt noch zu erledigen; nichtsdestoweniger wird der Berufung an den Kaiser ohne alles Bedenken Raum gegeben, und die Fortsetzung des Verfahrens einstweilen eingestellt. (Vgl. Planck, Bew.-Urth. p. 106 Anm. 1.) Wenn hiernach zweifellos dargethan ist, dass zu jener Zeit auch Zwischenurtheile durch Berufung an den höheren Richter angefochten werden durften, so bleibt es doch fraglich, ob gleicherweise in dem regelmässigen Processe von dem durch die Formel bestellten iudex privatus derartige appellable Interlocute erlassen werden konnten. Die L. 39 cit. redet nämlich von in integrum restitutio, also einem Falle der extraordinaria cognitio; ebenso ist in L. 4 C. de sent. quae sine cert. quant. VII, 46 mit ausdrücklichen Worten gesagt, dass das dort erwähnte Interlocut von einem Richter, qui extra ordinem iudicabat, gesprochen sei, und aus

L. 2 D. de app. recip. 49, 5. Ante sententiam appellari potest, si quaestionem in civili negotio habendam iudex interlocutus sit, vel in criminali, si contra leges hoc faciat (Scaevol. lib. 4 Regul.)

könnte man argumento a contrario sogar entnehmen, wenn man die Stelle überhaupt auf den ordentlichen Civilprocess bezieht, dass nur in dem dort genannten Falle die Appellation gegen ein Interlocut des Judex zulässig sei. Mindestens 
ist dies Fragment durchaus ungeeignet, den Beweis zu erbringen, dass die Zwischenbescheide des Richters im Formularverfahren regelmässig im Wege der Appellation anfechtbar waren.

Nun hat aber schon Cujacius a. a. 0. (0. 0. Neap. 1722 Tom. X p. 549) unter Berufung auf L. 38 pr. D. de minor. 4,4 , wo der Prätor als untere, der praefectus urbi als 7 weite Instanz auftreten, bemerkt, dass in unserem $\S 5$ unter dem competens iudex eben der praef. urbi, unter dem iudex tutelae - denn so verbindet Cujacius die Worte - der praetor tutelaris zu verstehen sei (vgl. auch Mommsen, Staatsrecht Bd. II p. 945 Anm. 3), und Gradenwitz (in dieser Zeitschrift Bd. VII p. 64) erblickt in dem competens iudex sogar eine Justinianische Interpolation. Man mag ihm darin beistimmen, obschon nicht recht abzusehen ist, weshalb die Compilatoren in dem einen Fragmente abändern, was sie in dem anderen, der L. 38 pr. cit. ungeändert haben stehen lassen. Ob auch die Worte "apud iudicem tutelae egit" auf einer Interpolation beruhen, darüber spricht sich Gradenwitz selbst mehr zweifelnd aus. Zieht man, was sprachlich am nächsten liegt, tutelae zu egit, so erscheint apud iudicem nichtssagend und selbst ungenau, man müsste denn eine concurrirende Gerichtsbarkeit des iudex mit einer sonstigen Behörde, etwa dem praetor tutelaris, annehmen. Verbindet man apud iudicem tutelae, so kann man allenfalls durch den Hinweis auf den iudex tutelaris bei Paul. R. S. V, $16 \S 2$ den iudex tutelae in L. 122 $\S 5$ D. V. O. zu halten versucht sein, jedoch steht dann wieder das egit in auffallender Weise isolirt, wiewohl bei dieser Bezeichnung des Richters kein Zweifel obwalten kann, dass eben die actio tutelae gemeint ist. Man kann eben nur so viel sagen, eine gewisse Wahrscheinlichkeit liegt vor, dass auch bei diesen Worten der ursprüngliche Text absichtlich geändert oder zufällig verderbt sei. Wie man nun aber auch darüber denken mag, dass das Verfahren vor dem praetor tutelaris in den ihm überwiesenen Cognitionen über die Bestellung, Excusation und Remotion von Vormündern das der extraordinaria cognitio war, steht ausser allem Zweifel. Gilt jedoch das Gleiche hinsichtlich der actio tutelae? Ja, gehörte diese Klage überhaupt vor sein Forum, war er dafür ausschliesslich 
oder neben dem regelmässigen Gerichte competent? Heisst iudex tutelae und iudex tutelaris in den angezogenen Stellen (vgl. auch Rudorff, Vormundschaft Bd. I p. 346 Anm. 38) nicht vielleicht bloss ,der für die Vormundschaftsklage bestellte iudex privatus" ohne alle Beziehung auf den praetor tutelaris? Hierfür lässt sich einmal der Bericht des Julius Capitolinus vit. Marc. c. $10 \S 11$ : „Praetorem tutelarem primus fecit, cum ante tutores a consulibus (cf. Suet. Claud. c. 23 Plin. ep. IX, $13 \S 16$ ) poscerentur, ut diligentius de tutoribus tractaretur" anführen. Der Schriftsteller beschränkt augenscheinlich den Geschäftskreis der neu creirten Magistratur auf die Bestellung der Vormünder und was damit zusammenhängt. Dann ist in den uns erhaltenen Fragmenten aus Ulpians und Paulus' liber singularis de officio praetoris tutelaris (L. 3. 5. 9, L. 6 \$ 13. 19 D. de excus. 27, 1 fr. Vat. $\S 232-236,238-242$. 244. 245. 247) überall nur von Vormundsbestellung und Excusation, nirgends von Verhältnissen die Rede, die mit der Vormundschaftsklage in $\mathrm{Zu}$ sammenhang gebracht werden könnten. Endlich ist die Competenz zur Ernennung u. s. w. von Vormündern bekanntlich von Alters her eine von der regelmässigen iurisdictio völlig gesonderte gewesen (L. 6 \& 2 D. de tutel. 26, 1, L. 8 pr. D. de tut. dat. 26,5), und so natürlich es auf den ersten Blick erscheinen mag; die Entscheidung über die gegenseitigen Ansprüche aus der Verwaltung der Vormundschaft in dieselbe Hand zu legen, welcher die Ernennung der Vormünder anvertraut ist, m. a. W. die actio tutelae gleichfalls dem praetor tutelaris zuzuweisen, so haben wir doch nicht den mindesten Anhalt dafür, dass dies bei den Römern wirklich geschehen sei.

Dennoch zeigt das Verfahren bei der actio tutelae im Vergleich mit den übrigen Civilklagen einzelne Besonderheiten. In Veranlassung eines Decretes des Kaisers Severus war dem Richter zum Zwecke der Prüfung der seitens der Vormünder geführten Verwaltung des Vermögens der Mündel gestattet worden, in Ermangelung anderer Documente die von den Sklaven angefertigten Rechnungen einzufordern und durchzusehen, und daran hatte sich dann die Möglichkeit einer quaestio de servis angeschlossen; ein Vorgehen, welches ursprünglich keineswegs in der Machtvollkommenheit des iudex 
privatus gelegen hatte, und welches Ulpian in L. 1 \$ 3 D. de act. tut. 27, 3 wesentlich nur durch die Berufung auf die üblich gewordene Praxis rechtfertigt: „de servis quoque interrogationes, sed et quaestiones habendas et hoc officio iudicis convenire placuit". Dabei sollte nicht ohne weiteres zur Folterung der Sklaven geschritten werden, sondern, wie der Schluss des Paragraphen besagt, erst dann, wenn die Sklaven von der Gegenpartei einer Fälschung der Rechnungen bezichtigt wurden. Die gleiche Ausnahmestellung der actio tutelae bezeugt

Paul. R.S. V, 16 \& 2. Iudex tutelaris itemque centumviri, si aliter de rebus hereditariis vel de fide generis instrui non possunt, poterunt de servis hereditariis habere quaestionem.

Man wende nicht ein, dass der unmittelbar voraufgehende Satz diese Befugniss dem Richter auch in anderen Civilklagen gewähre, sobald es sich um eine eigene Handlung des Sklaven fragt. Dort ist nämlich nur von dem Rechte zur interrogatio die Rede; interrogare und quaestionem habere sind aber, wie eben L. 1 \& 3 D. de tut. act. zeigt (vgl. auch L. 9 \& 1 D. de quaest. 48,18, L. $58 \S 2$ D. de aedil. ed. 21,1, L. 6 C. de quaest. IX, 41), durchaus nicht gleichbedeutend. Und wenn Aquila in L. 34 D. de adm. tut. 26, 7 mit Beziehung auf die Vormundschaftsklage bloss von der interrogatio spricht, so erklärt sich das daraus, dass es sich in dem ihm zur Begutachtung vorgelegten Falle, wenigstens zunächst nur um das Verhör, nicht um die Tortur der Sklaven handelte. In L. 9 pr. D. de quaest. legt Marcian augenscheinlich das Hauptgewicht darauf, dass in Civilsachen die Folterung der Sklaven nur ganz ausnahmsweise stattfinden dürfe. Allerdings ist wohl das dort angezogene Rescript des Antoninus Pius ursprünglich weiter gegangen, nicht sowohl in Betreff der speciellen Voraussetzung für die Zulässigkeit der quaestio, denn „si aliter veritas inveniri non possit" war auch da gesagt, als hinsichtlich der Gattungen von Rechtsstreitigkeiten, im Rescript hiess es schlechtweg ,in pecuniaria causa", bei denen eine quaestio statthaben durfte. Allein dem trat alsbald eine beschränkende Praxis entgegen, die in den Verordnungen des Severus bekräftigt wurde, und als deren Resultat wir den in 
der ausgeschriebenen Sentenz des Paulus angegebenen Rechtszustand zu betrachten haben. - Dass ferner das auf Vornahme der quaestio lautende Zwischenurtheil des iudex, weil seine Ausführung nicht wohl ohne wesentliche Schädigung der Interessenten möglich war, der Appellation unterlag, haben wir schon aus L. 2 D. de app. recip. ersehen. Liess man aber einmal die Berufung gegen diese Interlocute $\mathrm{zu}$, so war es kaum zu vermeiden, dass sich dieselbe auch anderen Zwischenbescheiden gegenüber, welche für den Ausgang des ganzen Processes präjudicirlich waren, nach und nach einbürgerte. Wir dürfen nach alledem für die Zeit des Scävola die Appellabilität der Interlocute, wenn nicht in weiterem Umfange, so doch jedenfalls bezuiglich der actio tutelae, auch da, wo diese Klage mittelst des Formularverfahrens zum Austrage gebracht.wurde, ohne Bedenken annehmen, und in dem so gestalteten Processe eine Uebergangsform zwischen jenem und der extraordinaria cognitio erblicken. Vielleicht hängt es mit dieser Entwickelung der Vormundschaftsklage zusammen, dass sich in den betreffenden Titeln der Justinianischen Compilation so gut wie gar kein Material zur Reconstruction der im Edict für die actio tutelae aufgestellten Formel erhalten hat. Somit erscheint es nicht unberechtigt, die in L. 122 $\S 5$ D. V. O. erwähnte Appellation als eine solche gegen ein Zwischenurtheil anzusprechen. Die in der Stelle selbst angegebenen Momente weisen darauf hin, und die allgemeinen processualischen Grundsätze stehen hier wenigstens nicht im Wege.

Allein es bleibt noch ein Bedenken zu erledigen übrig. Musste die Berufung auf den abgeschlossenen Vergleich, wenn dieser für rechtsbeständig erklärt war, nicht die Abweisung des Klägers zur Folge haben? Hatte die Verhandlung als extraordinaria cognitio stattgefunden, so war das sicherlich nicht geboten. Beklagte räumte ja ein, aus der Vormundschaft auf Grund des Vergleiches noch eine bestimmte Summe schuldig zu sein, der erkennende Richter war also nicht gehindert, sie darauf zu verurtheilen. Nicht anders steht es, wenn die Einrede des Transacts nur in der Gestalt der allgemeinen exceptio doli, quae bonae fidei iudiciis inest, geltend gemacht, nicht aber als ausdrückliche exceptio der Formel in- 
serirt wurde. Denn von einer Novation konnte bei der Formlosigkeit des Vergleichs keine Rede sein, und auch die über die Restsumme abgeschlossene Stipulation änderte daran nichts. Allein wenn nun wegen des Vergleiches eine ausdrückliche exceptio in die Formel aufgenommen war? Möglich, dass der Prätor dieselbe unter solchen Umständen als übertlüssig überhaupt nicht zuliess. Wo dies aber dennoch geschah, konnte sie nur als exceptio condemnationem minuens wirksam werden. Sehen wir nämlich von der Stipulation auf den Rest der Vergleichssumme einstweilen ab, so blieb, wenn der Gegner nicht freiwillig leistete, dem Erben des Pupillen durchaus keine Wahl; er hatte nur die actio tutelae, un seine Anspriche wahrzunehmen; auch kann er sich bei der incerta intentio der Klage keiner pluris petitio schuldig machen (Gai. IV § 54), seine Abweisung ist also unmöglich. Sie konnte aber auch nicht deshalb erfolgen, um ihn auf die Stipulationsklage $z u$ verweisen, da es ungereimt ist, einen zweiten Process zu provociren, wo man mit dem ersten zum Ziele gelangen kann; auch deckte sich, wie eben die Frage nach etwaigen Verzugszinsen in unserem Paragraphen zeigt, das Object in den beiden Processen nicht vollständig.

Die sachliche Entscheidung in unserem Paragraphen macht keine Schwierigkeiten. Scävola steht anscheinend noch ganz auf dem Standpunkte des Marcellus in L. $28 \S 1 \mathrm{D}$. de adm. tut. 26, 7. Erst durch eine Reihe von Constitutionen des Severus (cf. L. $26 \S 1$ D. de fideic. lib. 40, 5) ist der Begriff der mora ex re zu Gunsten der Minderjährigen, deren Folgen in innerlich durchaus consequenter Weise nur durch Deposition, nicht durch Oblation der schuldigen Leistung abgewendet werden konnten, in der römischen Jurisprudenz zur allgemeinen Anerkennung gelangt. Daher die verbessernde Note des Ulpian zu Marcellus a. a. O. Vgl. auch Papinian in L. $1 \S 3 \mathrm{D}$. de usur. 22, 1. Diese Theorie ist denn auch die des Justinianischen Rechts. Auf die Erben der Minderjährigen sollte davon aber keine Anwendung gemacht werden. Das zeigt auf das deutlichste die Einschiebung der L. 29 D. de adm. tut. hinter die Note des Ulpian. So konnte denn auch das Responsum des Scävola, wonach es für den besprochenen Fall einzig auf die gewöhnliche Mora ex persona 
ankommt, die durch einfache Oblation ausgeschlossen wird, unverändert in Justinians Digesten aufgenommen worden.

2. L. 35 pr. D. de donat. 39, 5. Ad eum, quem manumiserat, epistulam misit in haec verba: „Titius Sticho liberto suo salutem. cum te manumiserim, peculium quoque tuum omne, quidquid habes tam in nominibus quam in rebus moventibus sive in numerato, me tibi concedere hac epistula manu mea scripta notum tibi facio." eundem libertum testamento ex besse scripsit heredem, Sempronium ex triente: nec peculium Sticho legavit nec actiones praestari iussit. quaesitum est, utrum in assem Sticho actio detur eorundem nominum, quae in peculio habuit, an utrisque heredibus pro portionibus hereditariis. respondit secundum ea quae proponerentur utrisque heredibus pro hereditariis portionibus competere. (Scaevola Lib. 31 Dig.)

Der bereits früher (Bd. XI dieser Ztschr. p. 84 ff.) erklärte $\$ 1$ dieses Fragmentes fülurte uns schon direct in das Recht der lex Cincia hinein. Natürlich wird auch der vorliegende Rechtsfall von den Bestimmungen dieses Gesetzes beherrscht, und es ist deshalb die erste Frage, die wir uns vorzulegen haben, die, ob der Freigelassene seinem Patron gegenüber zu den personae exceptae gehöre. Noch neuerdings ist dies von Huschke behauptet worden. Dass der Freigelassene im Verhältniss zur Patrona keine solche Ausnahmestellung einnimmt, ergiebt sich allerdings aus fr. Vat. $\S 313$ und wird auch von Huschke ohne weiteres eingeräumt (Iurispr. anteiust. ed. V p. 805 not. 7). Indessen wäre es nicht gerade befremdlich, wenn man den patronus und die patrona in dieser Beziehung nicht gleich behandelte; man konnte es ganz angemessen finden, dass der Freigelassene in dem Patron zugleich eine wirthschaftliche Stütze hatte, während man ein derartiges Verhältniss zur patrona wenigstens nicht gesetzlich begünstigen mochte. Es kommt hier alles darauf an, wie man den Text von $\S \S 307$ und 309 der Vat. Fragm. gestaltet. Huschkes Reconstruction der beiden Paragraphen ist nun auf alle Fälle unhaltbar; liess sich das Gesetz so direct über den fraglichen Punkt aus, wie es das duitve is bei Huschke thut, so konnte die ganze Frage unmöglich, wie das in $\S 309$ ge- 
schieht, als eine zweifelhafte, nur durch die Praxis zu erledigende behandelt werden. Es ist vielmehr mit Mommsen in seiner Ausgabe der fr. Vat. anzunehmen, dass der Freigelassene auch seinem patronus gegenüber unter den Beschränkungen der lex Cincia stand. - Für die Ueberlassung des Peculiums macht es nun bezüglich der dazu gehörigen Sachen keinen Unterschied, ob der Beschenkte persona excepta ist oder nicht. Im einen wie im anderen Falle werden die Peculiarsachen pro donato resp. pro suo von ihm ersessen fr. Vat. $\$ 260$. 261. Wie steht es aber mit den Peculiarforderungen? Nicht schon in der blossen concessio peculii, d. $h$. hier dem non adimere peculium an sich, liegt die Berechtigung zur Anstellung der Peculiarklagen. Auch der emancipirte Sohn, die von der Mutter beschenkte Tochter werden erst vermöge einer Delegation oder lite inchoata als cognitores in rem suam klagberechtigt (fr. Vat. $\S 260.263$ ), und dem völlig entsprechend sagt Paulus in L. 53 D. de pecul. 15, 1, dass der Freigelassene, dem sein Peculium nicht entzogen sei, nur mandatis actionibus die Peculiarforderungen eintreiben könne. Es ist also in keiner Weise die lex Cincia, die in dem vorliegenden Rechtsfalle einen massgebenden Einfluss auf die Entscheidung äussert, sondern einzig der Mangel einer Universalsuccession in das Peculium und die daraus sich ergebende Nothwendigkeit eines besonderen Cessionsactes, der in dem blossen Schweigen und Belassen des Sondergutes nicht zum Ausdruck kommt, während bezüglich der Peculiarsachen das factische Behalten derselben und die dadurch bedingte Aenderung der Besitzverhältnisse als eine Reihe von Acten des Einzelerwerbes erscheint. Könnte noch irgend ein Zweifel darüber obwalten, dass die Bestimmungen der lex Cincia hierbei ganz einflusslos sind, so müsste er für unsere Stelle aus dem Grunde hinfällig werden, dass der Schenker bereits verstorben ist, und morte Cincia removetur, ein Satz, der bekanntlich nicht bloss von Sachschenkungen, sondern ebenso von obligatorischen Schenkungen gilt (fr. Vat. § 263. 266. 312). Wir dürfen uns deshalb auch der schwierigen und bei dem Stande unserer Quellen kaum zu einem befriedigenden Resultate führenden Untersuchung überheben, ob für die durch Delegation und Klagencession vollzogenen 
Schenkungen zwischen den personae exceptae und non exceptae etwa darin ein Unterschied bestand, dass letztere, nicht aber die ersteren einer actio rescissoria oder einer Condiction des empfangenen Werthes seitens des Schenkers unterlagen. Man könnte sich nämlich vielleicht versucht fühlen, durch eine derartige Unterstellung den in die Augen fallenden Widerspruch zwischen L. $2 \S 1.2$, L. $33 \S 3$ D. de donat., wo die Schenkung nach bewirkter Delegation als perfecta bezeichnet wird, einerseits und L. 21 § 1 D. eod. sowie L. 5 \& D. de dol. m. exc. 44,4 , wo unter anscheinend völlig gleichen Voraussetzungen condictio und actio rescissoria gegeben werden, andererseits, für das vorjustinianische Recht heben zu wollen. In Betreff der Justinianischen Compilation hilft die Annahme, dass in L. $2 \S 1.2$ und L. $33 \S 3$ D. de donat. keine insinuationspflichtige Summe in Frage stehe; denn in den beiden anderen Fragmenten ist, und, wenn ich nicht sehr irre, durch die Compilatoren, mittelst der Worte immodicae und supra legitimum modum (wegen dieser Interpolation vgl. L. 5 \$ 2 D. cit., wo Paulus ersichtlich von der exceptio legis Cinciae gesprochen hat) die Schenkung ausdrücklich als übermässige bezeichnet. Allerdings ist in L. 21 \& 1 D. cit. gegen den Schluss die weitere Interpolation mit besonderem Geschick gemacht. Dagegen dïrfte es ziemlich unmöglich sein, in L. $2 \S 1.2$ und L. $33 \S 3$ D. cit. eine Beziehung auf personae exceptae hineinzuinterpretiren, und ebensowenig ist dort an eine geringere Summe unter dem Mass der lex Cincia zu denken. Regelmässig nämlich setzen die Juristen, wo sie die Gültigkeit einer Schenkung mit Ruicksicht auf das Gesetz behandeln, die Ueberschreitung des Masses stillschweigend voraus. Es hängt das damit zusammen, dass in der lex, abgesehen von den besonderen Bestimmungen über Geschenke an Sachwalter, bloss das aligemeine Schenkungsverbot über das Mass und die Einzelanordnungen hinsichtlich der personae exceptae enthalten waren, wogegen die Feststellung der besonderen Formen, die nothwendig sind, um die Schenkung dem Einflusse der lex Cincia zu entziehen, sowie des Satzes „morte Cincia removetur" durch die Jurisprudenz bewirkt ist. Von letzterem wird uns das eigentlich in fr. Vat. § 266 mit klaren Worten gesagt, aber auch für die Formerfordernisse ist das gleiche aus 
der bloss indirecten Art ihrer Geltendmachung, aus der Möglichkeit einer Schulcontroverse (fr. Vat. \$ 266) über die Tragweite der exceptio legis. Cinciae, endlich aus dem Umstande mit Sicherheit zu folgern, dass unsere Quellen nirgends diese Formen aus der positiven Gesetzesvorschrift ableiten, so nahe auch der Anlass dazu gelegen hätte. Es ist mir völlig unverständlich, wie Puchta (Instit. $\S 206$ Anm. aa) aus fr. Vat. $\S 259$ und 310 entnehmen will, dass die lex Cincia eine bestimmte Form vorgeschrieben habe. Denn wenn es hier heisst, die Schenkungen seien gültig, weil ihnen unerachtet ihrer Formlosigkeit die exceptio legis Cinciae nicht entgegenstehe, so ist damit doch nicht mehr gesagt, als dass diese Einrede im übrigen der Gültigkeit formloser Schenkungen im Wege steht, und man übersieht ganz, wie die Beobachtung der Form einzig darin wirkt, dass für eine exceptio überhaupt kein Raum mehr vorhanden ist, und deshalb auch von der exceptio legis Cinciae kein Gebrauch gemacht werden kann. Nach alledem ist es verständlich, wie mit dem Aufwerfen der Frage, ob die lex Cincia Platz greife, schon die Thatsache ausgesprochen ist, dass eine Ueberschreitung des gesetzlichen Masses stattgefunden habe, ohne dass dies noch ausdrücklich wiederholt $\mathrm{zu}$ werden braucht. Ich wüsste nur eine Stelle, die L. 24 D. de donat., wo die Erwähnung des Uebermasses vielleicht echt ist, und der Jurist die Worte „supra modum legis" deshalb eingefügt haben mag, um die im Folgenden genannte exceptio als die exc. 1. C. zu charakterisiren; ausgeschlossen ist aber auch hier die Interpolation nicht. Javolenus kann sehr wohl die Einrede direct genannt haben, was dann die Compilatoren zu einer Textänderung veranlassen musste (vgl. auch Puchta a. a. O. Ann. q). Bei L. $21 \S 1$ D. cit. kommt ausserdem als Grund für das Vorhandensein einer Interpolation neben dem aussergewöhnlichen Gebrauche des ,immorlicus" namentlich noch hinzu die bloss theilweise Ungültjgkeit der Schenkung über das gesetzliche Mass, welche dem Justinianischen Rechte allerdings ganz entspricht (L. 34 C. de donat. VIII, 54), wovon uns aber in der classischen Zeit sonst keine Spur begegnet. Die entgegengesetzte Behauptung Rudorffs (Röm. RG. I $\S 22$ Anm. 11), die exceptio legis Cinciae sei minuendae condemnationis, ist völlig will- 
kürlich und wird durch die Berufung auf fr. Vat. \$ 294 in keiner Weise unterstützt. Die Worte ,quoniam quod praecessit totum irritum est" bezeichnen die totale Nichtigkeit des voraufgegangenen Actes im Gegensatz zu der nur durch Einrede geltend zu machenden Ungültigkeit der Schenkung contra legem Cinciam und die in Folge davon unmögliche Convalescenz des ersteren. (Vgl. auch Puchta. Inst. $§ 206$ Anm. nn; Schilling, Inst. Bd. 1 II p. 885 Anm. pp.) Der Versuch, die L. $2 \S 1.2$ D. h. t. schon im Sinne Julians auf eine Schenkung unter dem Masse des Cincischen Gesetzes zu deuten, muss hiernach wohl aufgegeben werden. Auch damit ist die Schwierigkeit nicht $z u$ heben, dass man sagt, der Jurist habe in der Stelle von diesem Gesetze ganz abgesehen und nur von dem geredet, was im übrigen zum Zustandekommen der Schenkung erforderlich sei (v. Meyerfeld, Schenkung $\$ 15$ p. 261). Es ist ja ganz richtig, dass donatio perfecta keineswegs allein mit directer Beziehung auf die Vorschriften der lex Cincia gebraucht wird. So findet sich non perfocta donatio in fr. Vat. $\S 257$, wo das Zustandekommen jeglicher Schenkung geleugnet werden soll, ähnlich in $\S 268, \mathrm{~L} .11 \mathrm{C}$. de donat. VIII, 54 und umgekehrt perficere donationem in Fällen, bei denen die lex Cincia, weil es sich um personae exceptae handelt, u. s. w. gar nicht in Anwendung kommen kann (fr. Vat. $\S 255$. 264. 278). Allein es erscheint kaum möglich, eine Schenkung als perfecta zu bezeichnen, wenn sie noch nach der lex Cincia angefochten werden kann, denn dann ist sie eben noch nicht gültig zu Stande gekommen. Es war das um so weniger zulässig, als jener Ausdruck ja gerade in Beziehung auf das Gesetz beinahe $\mathrm{zu}$ einem typischen geworden ist. Ganz anders natürlich, wenn nachträglich andelweitige Gründe für die Aufhebung der bereits gültig vollzogenen Schenkung eintreten, wie Anstellung der querela inofficiosae donationis, Revocation der Schenkung durch den Patron u. s. w. Da nimmt man allerdings keinen Anstand, die der Art aufhebbare donatio als perfecta zu bezeichnen (fr. Vat. $\S$ 272. 293). Wir werden also einräumen müssen, dass für das classische Recht eine Vereinigung der einander widerstreitenden Fragmente L. $2 \S 1.2$, L. $33 \S 3$ D. de donat. und L. $21 \S 1$ D. eod., L. 5 \& 5 D. de dol. m. exc. 44,4 
nicht eben allzu leicht ist (vgl. auch Schlesinger, Formalcontr. p. 126. 127 Anm. 32). Am meisten hat vielleicht noch die Vermuthung für sich, dass hier eine Controverse zwischen dem Sabinianer Julian und dem Proculianer Celsus - und Paulus schliesst sich mit einer gewissen Vorliebe den proculianischen Ansichten vielfach an - hineinspiele.

- Es bleibt somit für unser Fragment nur noch die Frage zu beantworten, ob nicht das in L. 53 D. de pecul. 15, 1 und L. 2 C. de donat. VIII, 54 zur Vollziehung der Schenkung für ausreichend erklärte Klagemandat in dem mitgetheilten Briefe thatsächlich enthalten sei. Es wäre nun allerdings denkbar, dass Scävola dies Mandat als durch den Tod des Schenkers hinfällig geworden betrachtet und deshalb für die ungehinderte Vererbung der Peculiarforderungen entschieden hätte. Auf einen solchen Gedanken könnte allenfalls das von Justinian in L. $33 \mathrm{C}$. de donat. erwähnte und aufgehobene ältere Recht hinleiten. Allein ich glaube doch, eine andere, schon von dem Anonymus (Basil. Lib. 47 Tit. 1 c. 34 schol. 2 ed. Heimbach IV p. 579) gegebene Erklärung liegt hier näher. Ein Mandat zur Einziehung der Peculiarforderungen ist überall nicht ertheilt. Die Zuschrift des Patrons an den Freigelassenen enthält ein solches nicht, überhaupt liegt in derselben keinerlei Dispositionsact, sondern bloss eine Benachrichtigung über das, was der Freilasser gethan hat, nämlich über die mit der Freilassung verbundene vollständige concessio peculii, die zur Uebertragung der Peculiarklagen eben nicht genügt. Es steht mit der Epistel in unserem Fragmente einigermassen ähnlich wie mit der in L. 32 D. de donat. Auch diese enthält schon ihrer Form nach keine directe, rechtliche Anordnung; es wird darin weder eine Servitut bestellt, noch eine obligatorische Verpflichtung zu irgend welchem Leisten übernommen. Da lautet L. 27 D. eod. ,dono et permitto tibi habitare" doch schon anders. Ja, dass in L. 32 jeder juristische Zwang ausgeschlossen bleiben soll, sagt im Grunde die Clausel "idque te ex voluntate mea facere" geradezu, indem das dem Adressaten verstattete uti für seine gesammte zeitliche Ausdehnung auf die voluntas des Schreibenden gestiitzt und aus dieser gerechtfertigt wird. Dem entsprechend ist dann in dem Responsum des Scävola nur von einem mutare voluntatem 
der Erben die Rede. Es handelt sich hiernach in L. 32 cit. um ein einfaches precarium (s. auch Windscheid, Pand. $\$ 368$ Anm. 11). Mit der lex Cincia hat die Stelle absolut nichts zu thun.

3. L. 27 D. de probat. 22, 3. Qui testamentum faciebat ei qui usque ad certum modum capere potuerat legavit licitam quantitatem, deinde ita locutus est: „Titio centum do lego, quae mili pertulit: quae ideo ei non cavi, quod omnem fortunam et substantiam, si quam a matre susceperat, in sinu meo habui sine ulla cautione. item eidem Titio reddi et solvi volo de substantia mea centum quinquaginta, quae ego ex reditibus praediorum eius (quorum ipse fructum percepi et distraxi), item de calendario (si qua a matre receperat Titius) in rem meam converti." quaero, an Titius ea exigere potest. respondit, si Titius supra scripta ex ratione sua ad testatorem pervenisse probare potuerit, exigi: videtur enim eo, quod ille plus capere non poterat, in fraudem legis haec in testamento adiecisse. (Scaevola lib. $\mathbf{3 3}$ Dig.)

Die hier von Scävola über die Beweispflicht des Legatars getroffene Entscheidung will, von welcher Seite her man sie auch betrachten mag, mit den sonst geltenden Normen über die Vertheilung der Beweislast nicht ganz stimmen. Denn wenn auch der rein formale Gesichtspunkt, den Celsus in der L. 12 D. h. t. vertritt, der Legatar legt das Instrument mit der Anordnung zu seinen Gunsten vor, es ist nun die Sache des Gegners, dessen Unwirksamkeit darzuthun, in unserem Falle nicht geltend gemacht werden kann, da es bereits feststeht, dass das dem Titius zugewendete legatum debiti seiner Incapacität halber als Vermächtniss nicht zur Ausführung gelangen kann, so geräth das Responsum doch anscheinend in Conflict mit dem Princip der L. 18 \& 1 D. h. t.: „Qui dolo dicit factum aliquid, licet in exceptione, probare debet", welches Scävola selbst in L. 6 D. eod. anerkennt, indem er dem Patron, welcher die von dem Freigelassenen vorgenommene Veräusserung als betrüglich ansieht, den Beweis der betrüglichen Absicht auferlegt. Andererseits enthalten die Angaben des Testators ein Geständniss über eine ihm obliegende Schuld- 
verbindlichkeit, welches sonst, auch in Ermangelung jedes anderweitigen Beweises, als ausreichend zur Begründung einer Klage für den Gläubiger angesehen wird, freilich nicht in dem Sinne, als ob das Geständniss das Geschäft ersetze (vgl. auch L. $88 \S 10$ D. de leg. II, 31), wohl aber, soweit es sich um die Erbringung des Beweises über das Zustandekommen, die Existenz des Geschäfts handelt. L. $26 \S 2$ D. depos. 16, 3: ,ex epistula, de qua quaeritur, obligationem quidem nullam natam videri, sed probationem depositarum rerum impleri posse".

Man darf nun aber nicht ïbersehen, dass alle diese generellen Beweisregeln eben nur Regeln sein sollen, die für eine grosse Mehrzahl von Fällen allerdings zutreffen, die aber nicht den Charakter von unverbriichlichen Normen an sich tragen, wie etwa die Sätze der neueren gemeinrechtlichen Beweistheorie; vielmehr lassen sie unter geänderten Voraussetzungen sehr wohl Abweichungen zu. Das gilt schon von dem durch Celsus in L. 12 D. de prob. auf den von ihm behandelten besonderen Fall angewendeten Principe, wonach der Legatar, der zwei Urkunden, Testament und Codicill, mit gleichlautenden Zuwendungen an seine Person vorlegt, damit auch einen doppelten Anspruch dargethan hat, so dass es Sache des Gegners ist, den Nachweis zu führen, wie der Erblasser bei seinen gleichlautenden Verfügungen das Object des Legates (eine bestimmte Summe) nur einmal habe vermachen wollen. Ganz anders dagegen, wenn diese beiden Urkunden zwei Ausfertigungen desselben Testamentes sind, und in der einen eine Zuwendung enthalten ist, welche von der in der anderen bezüglich der genannten Summe abweicht. Hier wird, weil beide Documente sich als identische betrachtet wissen wollen, nur ein Vermächtniss und zwar das des geringeren Betrages angenommen (L. 47 D. de leg. II, 31). So ferner braucht der Gläubiger, welcher die Veräusserung des Schuldners als betrügerisch anfechten will, da den Beweis der fraus seinerseits nicht erst zu führen, wo sich letztere aus der nackten Thatsache unmittelbar ergiebt, wie bei einer Veräusserung des gesammten Vermögens (L. $17 \S 1$ D. quae in fraud. cred. 42,8), und umgekehrt beweist der mit der rei vindicatio Belangte, der seinen Besitz inzwischen verloren 
hat, in den Thatumständen, die diesen Verlust darthun sollen, zugleich, dass letzterer ohne bösliche Absicht von seiner Seite erfolgt ist (L. 17 D. de exc. rei iud. 44, 2). Hier also lässt die - eigenthümliche Verbindung der beweisenden Momente in ein und demselben Thatbestande eine Trennung der Beweisführung, wie sie den allgemeinen Regeln über die Vertheilung der Beweislast unter den Parteien entsprechen würde, gar nicht mehr zu. Und so ist endlich auch das gelegentlich eines anderweitigen Rechtsgeschäftes verlautbarte, aussergerichtliche Geständniss nicht immer und ohne weiteres glaubwürdig. Es kann dabei die Vermuthung begründet sein, dass die Abgabe des letzteren wesentlich deshalb erfolgte, um das gegenwärtig beabsichtigte Geschäft gegen diesen oder jenen Einwand zu sichern. Man kann hierher ziehen eine andere Stelle des Scävola: L. $37 \S 5$ D. de leg. III, 32. Dort handelt es sich wie in L. $26 \S 2$ D. depos. um ein Empfangsbekenntniss, aber Scävola sieht dasselbe, weil es in einem Codicill zur Begründung eines Vermächtnisses benutzt wird, anders wie Paulus, nur bedingungsweise als vollkräftig zum Erweise der geschehenen Deposition an. Man könnte den Grund für diese abweichende Auffassung der beiden Juristen in einem Umstande finden, auf den Bruns (Zeitschr. f. RG. I p. 127) in anderer Richtung Gewicht legt. Das Anerkenntniss, welches Scävola vorliegt, ist allerdings, mit Puchta zu reden, „eine entschieden einseitige Handlung", während in dem dem Gläubiger zugestellten Empfangsscheine, über den Paulus sich auslässt, gleichzeitig ein Vertragselement anzuerkennen ist. Allein wie sich aus der ganzen Argumentation des Scävola erselsen lässt, ist dies nicht der die Ansicht des Juristen bestimmende Punkt: ,scilicet cum iusiurandum dedisse super hoc testator affirmavit, credenda est scriptura". Unerachtet der Erwähnung des Eides bleibt die gesammte Aeusserung im Codicill doch durchaus einseitige Erklärung ohne eine Spur eines zweiseitigen Uebereinkommens. Es kann also nur die detaillirtere Angabe der begleitenden Umstände oder auch die Erwägung sein, der Testator werde nicht so leicht den Eid, als religiöse Handlung, zum Gegenstand einer Unwahrheit machen, was dem Schuldbekenntniss in den Augen des Juristen eine grössere Glaubwürdigkeit verleiht. Ich will 
nicht in Abrede stellen, dass der begründende Satz scilicet cum etc. einem Glossem einigermassen ähnlich sieht. Es könnte allenfalls eine von den Compilatoren in das Responsum hineingearbeitete Note des Claudius Tryphoninus sein, obgleich der eigenthümliche Anakoluth in der Antwort ihnen dann doch kaum entgangen sein könnte und von ihnen wohl vermieden worden wäre. Ich möchte deshalb eher, wie wir dergleichen ja bei Scävola gar nicht selten finden, eine Flüchtigkeit in der Redaction von Seiten des Autors selbst annehmen. Wie dem aber auch sei, jedenfalls würde dieser durch das beschränkende secundum ea quae proponerentur den Anlass zu der Ergänzung seiner Entscheidung gegeben haben, und es wäre nicht gerade unmöglich, dass dieselbe in einer ganz falschen Richtung erfolgt wäre. Jenes ,secundum ea quae proponerentur" sollte vielleicht nur sagen, "da nach dem Vortrage der Sachlage keinerlei Verdachtsmomente vorliegen", wie sie z. B. einem incapax gegenüber vorhanden sein würden. Eben deshalb legt der Jurist in unserer L. 27 D. de prob. dem Legatar ohne alle Rücksicht auf das Empfangsbekenntniss des Testators die Beweislast auf. Eine Parallelstelle dazu ist die L. 37 \& 6 D. de leg. III, 32, nur dass hier die in fraudem legis angeordnete Zuwendung nicht durch das Vorschützen eines in Wirklichkeit nicht bestehenden Schuldverhältnisses, sondern nur durch eine falsa demonstratio gerechtfertigt werden soll. Deshalb fehlt auch der Vorbehalt des Beweises für den Honorirten; es heisst einfach "non idcirco quod scriptum est exigi posse in fraudem legis relictum". Gewiss sollte damit dem Bedachten die Möglichkeit nicht abgeschnitten werden, einen ihm etwa geschuldeten Lohn einzuklagen resp. das Hinterlassene als wahres legatum debiti in Anspruch zu nehmen. Es lag nur bei der Ausdrucksweise der Erblasserin der Gedanke an das Bestehen einer solchen Schuld viel weniger nahe als in dem anderen Falle; daher das Schweigen über einen vielleicht doch zu erbringenden Beweis. In dieser Beziehung zeigt das Verhältniss dieser beiden Stellen eine gewisse Aehnlichkeit mit dem zweier anderen, die Paulus theilweise anführt.

L. 16 D. de dot. leg. 33, 4. Qui dotem a matre uxoris acceperat et stipulanti ei promiserat, testamento uxori 
dotem legavit. cum quaesitum esset, an uxor dotis summam consequi posset, respondit Scaevola non videri dari uxori, quod necesse sit matri reddi. alias sic respondit non videri, nisi manifeste uxor docuisset, eam testantis voluntatem fuisse, ut onerare heredes duplici praestatione dotis vellet. (Paul. lib. 2 ad Vitell.)

Auch hier sachliche Uebereinstimmung der beiden Responsen des Scävola, nur dass das eine auf die Zulässigkeit einer besonderen Beweisführung Rücksicht nimmt, worüber* das andere schweigt. Eben dieser Differenz wegen führt ja Paulus auch das zweite wörtlich an und bietet uns damit den vollen Beweis der Echtheit des beschränkenden Satzes „nisi manifeste uxor docuisset" etc. Damit aber erscheint zugleich der aprioristische Verdacht gegen die bei Scävola so häufig wiederkehrenden (vgl. diese Zeitschr. Bd. VIII p. 159 f.), in einem Zwischensatz mit nisi eingeführten Beweisvorbehalte entkräftet.

\section{III. \\ Custodia und vis maior. \\ Von \\ Herrn Dr. iur. Johannes Biermann \\ in Berlin.}

Franz Xaver Bruckner. Die custodia nehst ihrer Beziehung zur vis maior nach römischem Recht. Gekrönte Preisschrift der Universität München. München 1889.

Gotthold Gerth. Der Begriff der vis maior im römischen und Reichsrecht. Berlin 1890.

Zwei Erstlingsschriften liegen hier vor, beide sich fast auf dasselbe Thema beziehend, und jede $\mathrm{zu}$ einem Resultate gelangend, welches dem der anderen so ziemlich entgegengesetzt ist. Und wie die beiden Arbeiten im Resultate Gegensätze sind, so sind sie es auch in ihrem ganzen Habitus: das 